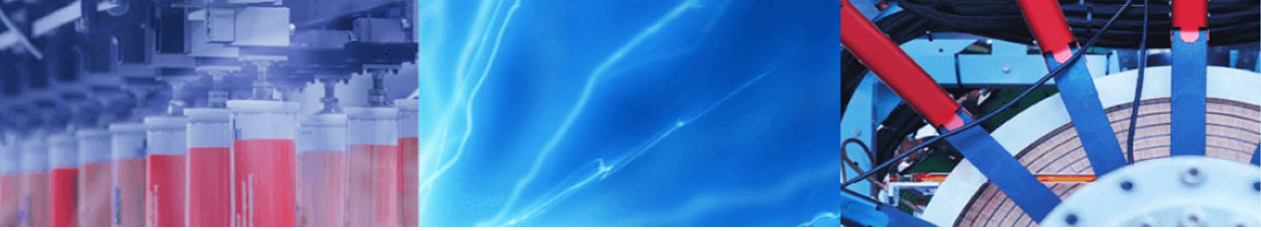

Research Article

\title{
Advanced chromatographic technique for performance simulation of anti-Alzheimer agent: an ensemble machine learning approach
}

\author{
U. M. Ghali ${ }^{1} \cdot$ Abdullahi Garba Usman $^{2}$ D $\cdot$ Z. M. Chellube ${ }^{3} \cdot$ Mohamed Alhosen Ali Degm $^{4} \cdot$ Kujtesa Hoti $^{5}$. \\ Huzaifah Umar6 . S. I. Abba ${ }^{7}$
}

Received: 22 July 2020 / Accepted: 13 October 2020 / Published online: 23 October 2020

(c) Springer Nature Switzerland AG 2020

\begin{abstract}
This work employs the application of three artificial intelligence (Al) techniques namely; support vector machine (SVM), Hammerstein-Wiener (HW) and multi-layer perceptron (MLP) for predicting the qualitative properties of an anti-Alzheimer agent using high-pressure liquid chromatography technique. The mobile phase (inform of acetonitrile and trifluoroacetic acid) and the column temperature was used as the predictors in modelling the maximum retention time (tR-max) and resolution (Resol.) as the output variables of the analyte. The measured and predicted values were checked using three performance indices including; Nash-Sutcliffe efficiency (NSE), correlation coefficient (CC) as the goodness of fits and a statistical error inform of root-mean-square error (RMSE). The results obtained demonstrated the promising ability of Al-based models in modelling the qualitative properties of the anti-Alzheimer agent. Observation of different outputs of the Al-based models at various time intervals showed the necessity of ensembling the outputs of the Al-based models. Therefore, simple average ensemble and support vector machine ensemble (SVM-E) were employed to enhance the performance skills of the simple models. The comparative performance of SVM-E inform of NSE indicated its ability in boosting and enhancing the performance skills of the single models SVM, MLP and HW models up to 5, 13 and 20\% respectively in the testing stage for modelling tR-max.
\end{abstract}

Keywords HPLC · Maximum retention time $(\mathrm{tR}-\mathrm{max}) \cdot$ Resolution · Anti-alzheimer agent · Ensemble machine learning · Artificial intelligence

\section{Introduction}

Generally, the most important part of optimization in chromatographic methods such as high-performance liquid chromatography (HPLC), gas chromatography (GC) and high-performance thin-layer chromatography (HPTLC) is the achievement of good resolution within short analysis time. In HPLC, this is adjusted using the gradient elution system, which is also known as a solvent programming system. This composed of changing the concentration/ composition of the mobile phase during the analysis to minimize the time of retention as well as to successfully separate the analytes [1].

The traditional optimization method involves the mathematical fitting of one variable at a time, while other variables are kept constant and fixed. This method consists of the optimization of $\mathrm{pH}$, which gives better selectivity due to the degree of ionization of the solutes, the mobile

Abdullahi Garba Usman, abdullahigusman@gmail.com | ${ }^{1}$ Faculty of Medicine, Department of Medical Biochemistry, Near East University, 99138 Nicosia, Turkish Republic of Northern Cyprus. ${ }^{2}$ Faculty of Pharmacy, Department of Analytical Chemistry, Near East University, 99138 Nicosia, Turkish Republic of Northern Cyprus. ${ }^{3}$ Department of Pure and Industrial Chemistry, University of Maiduguri, Maiduguri, Nigeria. ${ }^{4}$ National Centre for Diabetes and Endocrinology, Tripoli, Libya. ${ }^{5}$ Department of Technical Laboratory, College Heimer, Prishtina, Kosovo. ${ }^{6}$ Department of Bioengineering, Institute of Graduate Studies and Research, Cyprus International University, Mersin, Turkey. ${ }^{7}$ Department of Physical Planning Development, Maitama Sule University Kano, Kano, Nigeria. 
phase, and stationary phase additives, which might affect the $\mathrm{pH}$ [2]. It also focuses on optimizing the composition of the mobile phase, which is the ratio of the water and the organic modifier. Moreover, the optimization various chromatographic factors such as the composition of the mobile phase and column temperature are essential in obtaining sufficient resolution. Considering the high number of factors that influences separation, it is challenging as well as time-consuming in reaching the desired separation conditions, more especially employing the single parameter optimization method. Generally, this approach is tedious and time-consuming [3]. Therefore, to minimize these drawbacks scientists introduce a hyphenated process that involves the experimental and modelling method to achieve reasonable resolution within short analysis time. The use of Artificial Intelligence (AI) based models, and chemometrics has been reported in the technical literature for prediction in HPLC method development [4]. For example, Tham and Agatonovic-Kustrin reported the application of an artificial neural network (ANN) in the prediction of the retention behaviour of phenylthiocarbamyl amino acid derivatives coupled with a genetic algorithm. More also, Luan et al. also demonstrated the comparative use of a linear model heuristic method (HM) and non-linear model support vector machine (SVM) models for the qualitative prediction of different volatile compounds. The results demonstrated the reliability of SVM over the linear HM model [5]. D'Archivio [6] equally reported the application of ANN model in the prediction of retention factor of 16 different kinds of amino acids using reversed-phase liquid chromatography through applying different elution modes. The accuracy of ANN prediction was found to be far better than the previously obtained by fitting the same data with retention models based on the solution of the fundamental equation of gradient elution. This research explored the use of Humanin as well as its derivatives, which are considered as neural cells protecting agents against pathological proteins such as the amyloid protein precursor that causes Alzheimer's disorder. Alzheimer's disease is one of the neurodegenerative diseases that composed of cognitive disorders as well as dementia. The curing of this disease is of vital using agents such as Humanin and its derivatives [7]. Therefore, the analytical determination of Humanin and its derivatives is of paramount importance. Even though these agents have been studies and examined in the literature. Regarding the peptide bond separation, the reverse phase ion-pair high-pressure liquid chromatography (HPLC) with the used of trifluoroacetic acid (TFA) as the mobile phase is usually used to serve as an alternative over the ion-exchange liquid chromatography [8].

Based on the historical studies conducted in the technical literature, it can be observed that Al-based models are superior and promising predicting methods for modelling chemical processes [9].

However, some potential issues are attributed to $\mathrm{Al}$ models, such as generalization ability and overfitting. Therefore, scientist develops a new and advanced computational data-driven method, which can solve these potential problems. Ensemble data-driven method provides reliable performance skills as compared to that of the non-linear models. The novelty of this study is indicated in different forms; (1) since the introduction of the non-linear models, this is the first study in the technical literature depicting the applications of multi-layer perceptron (MLP), Hammerstein-Wiener (HW) and support vector machine for the prediction of humanin and its derivatives using HPLC technique. (2) To the best knowledge of the authors', this is the first study conducted in area of analytical chemistry depicting the application of support vector machine ensemble (SVM-E) using the chromatographic method. The major motivation of this study is to employ the applications of various Al-based models, such as multilayer perceptron (MLP), Hammerstein-Wiener (HW) and support vector machine (SVM) for predicting the maximum retention time (tRmax) and resolution of humanin as well as its derivatives in HPLC optimization method development using mobile phase inform of acetonitrile (ACN) and trifluoroacetic acid (TFA) and column temperature as the input parameters. Furthermore, to enhance and boost the performance of the MLP, HW and SVM models using simple average ensemble (SAE) as a linear machine learning technique and support vector machine ensemble (SVM-E) as a non-linear machine learning technique.

\section{Materials and method}

\subsection{Proposed methodology}

The understanding of data science is of paramount importance in any type of data driven technique, more especially in machine learning. In this work, three Al based models such as Multi-layer perceptron (MLP), Hammerstein-Wiener (HW) and support vector machine (SVM) model were used to predict the maximum retention time (tRmax) and resolution of humanin and its derivatives in HPLC optimization method development, the data was collected from Novotná et al. [7]. The mobile phase composition inform of acetonitrile (ACN) and trifluoroacetic acid (TFA) and column temperature are used as the input variables. Afterwards, simple average ensemble (SAE) and Support vector machine ensemble (SVM-E) were applied in order to enhance and increase the performance accuracy of humanin and its derivatives qualitative determination by uniting the output results of the MLP, HW and SVM models. One 
of the major motivation of employing different machine learning methods is the difficulty in determining whether a certain approach is superior to others using a certain data sets.

\subsection{Multi-layer perceptron (MLP) neural network}

One of the standard types of artificial neural network (ANN) is the multi-layer perceptron (MLP). MLP is a reliable tool that is capable of handling the non-linearity of a data set $[10,11]$. Just like other ANNs, the architecture of MLP consists of an output layer, a hidden layer and an input layer $[12,13]$. Generally, the signals are processed; they are transferred to the output layer via the input layer through sequential mathematical processes with the help of biases and weights. Generally, the Levenberg-Marquardt algorithm is used as the learning algorithm for optimizing the error that exists between the experimental and simulated values. The training algorithm is optimized until the desired result is found (Fig. 1) [14, 15].

$y_{i}=\sum_{j=1}^{N} w_{j i} x_{j}+w_{i 0}$

where $N$ is denoted as the sum of the number of the nodes, $i ; w_{j i}$ as the weight that exists between the nodes $i$ and $j ; x_{j}$ is denoted as the output which is accomplished from the node $j ; w_{i 0}$ as the bias in node $i$, and $y_{i}$ defines the input signal of node $i$ which crosses via the transfer function.

\subsubsection{Hammerstein-Wiener model (HW)}

Hammerstein-Wiener (HW) model composed of both linear and non-linear block systems, as shown in Fig. 2. HW model has the ability to be utilized in form of a black-box model. Whereby, it gives a flexible use of various parameters and
Fig. 1 Three-layer multi-layer perceptron structure
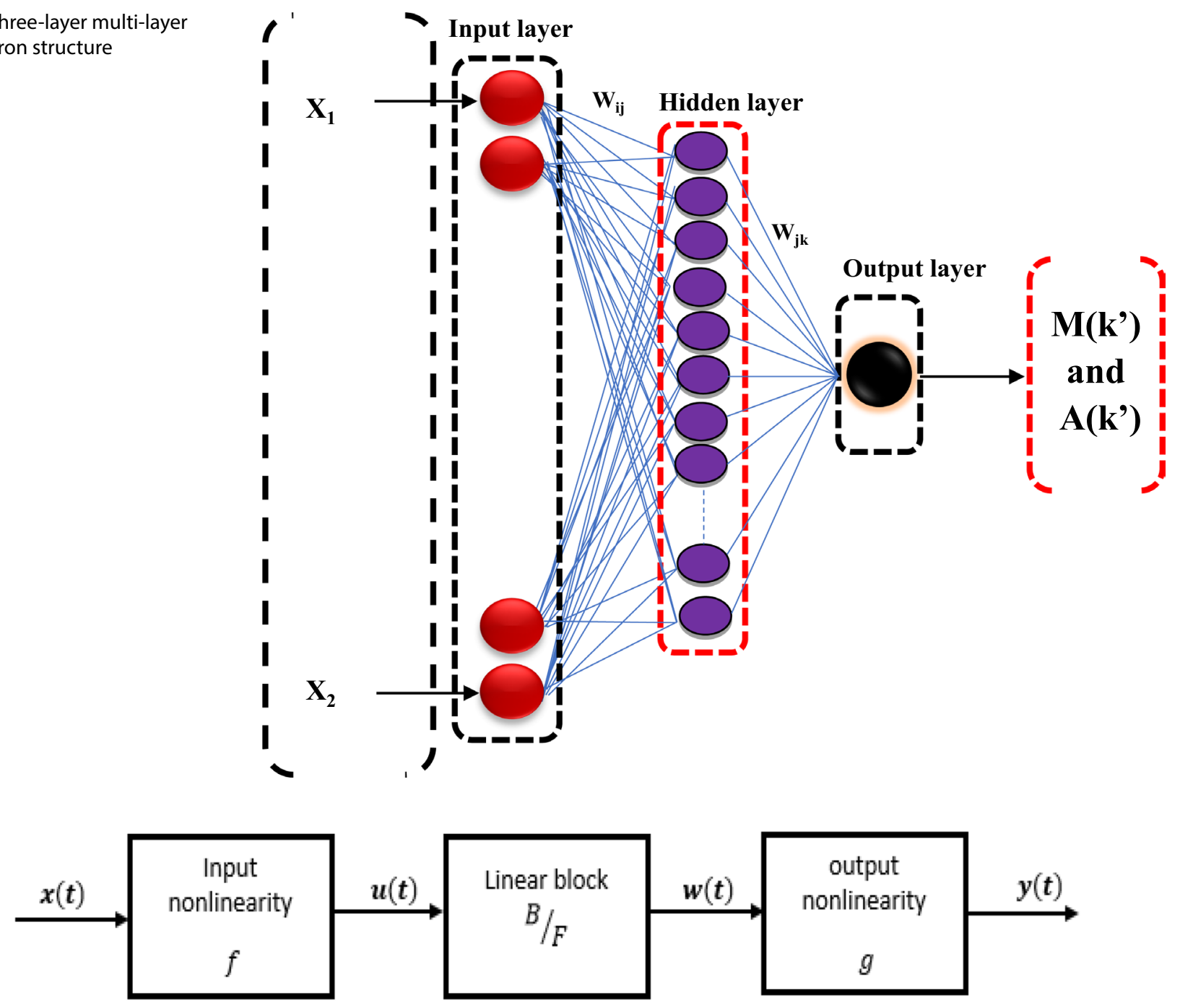

Fig. 2 Schematic of Hammerstein-Wiener model 
variables, and ensures that the basic and primary knowledge regarding the process of physical characteristics [16]. HW provides more satisfactory performance in various applications as compared to linear systems like the MLR and the non-linear systems such as ANN, since it considered the dual properties of the data set of both the non-linearity and linear properties of the data set [13]. The general structure of HW model composed of the application of a black-box process, which is developed purposely to predict the non-linearity [16]. The architecture of HW model consists of three blocks namely; static output non-linear blocks, a static input nonlinear block and a linear dynamic block $[17,18]$. HW mechanism involves the conversion of the nonlinear functions into linear input blocks, which are subsequently transferred back into the non-linear form again inform of output [17-19].

Where: $u(t)=f(x(t))$, is a non-linear function converting input data.

$w(t)=u(t)^{B} / F$, represent linear function, $f$ and $\mathrm{h}$ act on the input and output data of the linear block respectively.

The function $u(t)$ and $w(t)$ are variables that define the input and output of the linear block.

\subsubsection{Support vector machine (SVM)}

Vapnik developed the idea of support vector machine (SVM) in 1995. The developed idea was interested in resolving issues involving regression, pattern recognition, classification and prediction. The concept of SVM is similar to other machine learning algorithms that involved the development of data driven models [20]. SVM has to main functions, including; structural risk minimization and statistical learning theory. It has special features that differentiates it from other Al-based models like ANN i.e. ability to minimize error, complexity, redundancy of the data as well as increase the performance skills of the system. This model can be further categorized into; non-linear support vector machine and linear support vector regression [21].

The training data set is denoted as $\left\{\left(x_{i}, d_{i}\right)\right\}_{i}^{N}\left(x_{i}\right.$ is defines as input vector, $d_{i}$ is the experimental value and $N$ is total number of the instances), and the general SVM equation is denoted as:

$y=f\{x\}=w \varphi\left(x_{i}\right)+b$

where $\varphi\left(x_{i}\right)$ indicates feature spaces, non-linearly mapped from input vector $x$.

Minimize : $\frac{1}{2}\|w\|^{2}+C\left(\sum_{i}^{N}\left(\xi_{i}+\xi_{i}^{*}\right)\right)$
Subject to : $\left\{\begin{array}{c}w_{i} \varphi\left(x_{i}\right)+b_{i}-d_{i} \leq \varepsilon+\xi_{i}^{*} \\ d_{i}-w_{i} \varphi\left(x_{i}\right)+b_{i} \leq \varepsilon+\xi_{i}^{*} \\ \xi_{i}, \xi_{i}^{*}\end{array} \quad i=1,2, \ldots, N\right.$

where $\frac{1}{2}\|w\|^{2}$ are the weights vector norm and $C$ is referred to the regularised constant. The general conceptual model structure of SVM is illustrated in Fig. 5. The parameters of the Lagrange multipliers are defined as $a_{i}$ and $a_{i}^{*}$. Vector $w$ in Eq. (2) can be calculated after finding the problem solution of optimization.

$w^{*}=\sum_{i=1}^{N}\left(\alpha_{i}-\alpha_{i}^{*}\right) \varphi\left(x_{i}\right)$

Therefore, the overall form of SVM informs Eq. (6).

$f\left(x, \alpha_{i}, \alpha_{i}^{*}\right)=\sum_{i=1}^{N}\left(\alpha_{i}-\alpha_{i}^{*}\right) K\left(x, x_{i}\right)+b$

where $K\left(x_{i}, x_{j}\right)$ is the kernel function, and $b$ is the bias term. The radial basis function (Gaussian) is the most common kernel function and is expressed as:

$K\left(x_{1}, x_{2}\right)=\exp \left(-\gamma\left\|x_{1}-x_{2}\right\|^{2}\right)$

where $\gamma$ is the kernel parameter. Figure 3 shows the general structure of SVM model.

\subsection{Ensemble techniques}

It is evident that simple non-linear models provide various levels of accuracy for the same input variables based on their robustness and limitations. Thus, ensemble machine learning is applied in different field of science, engineering, social and management science and health science etc. In chemistry, it can be used in synthesis, spectroscopy, colour, polymer and chromatography [22-25]. Ensemble technique is collectively considered as a class of machine learning, which deals with compound heterogeneous and homogenous models [22]. The ensemble technique is used in order to boost the final performance skills of the simple models by combining their respective outputs as the new input variables. Various studies has proved the reliability of Ensemble techniques over the simple models in solving complex problems. In this work, two ensemble methods were employed to improve the performance prediction skills of the single models including; Simple Averaging Ensemble (SAE) and Support vector machine ensemble (SVM-E) through combining the outputs of HW, MPL and SVM single models. 
Fig. 3 The architecture of SVM Algorithms [21]

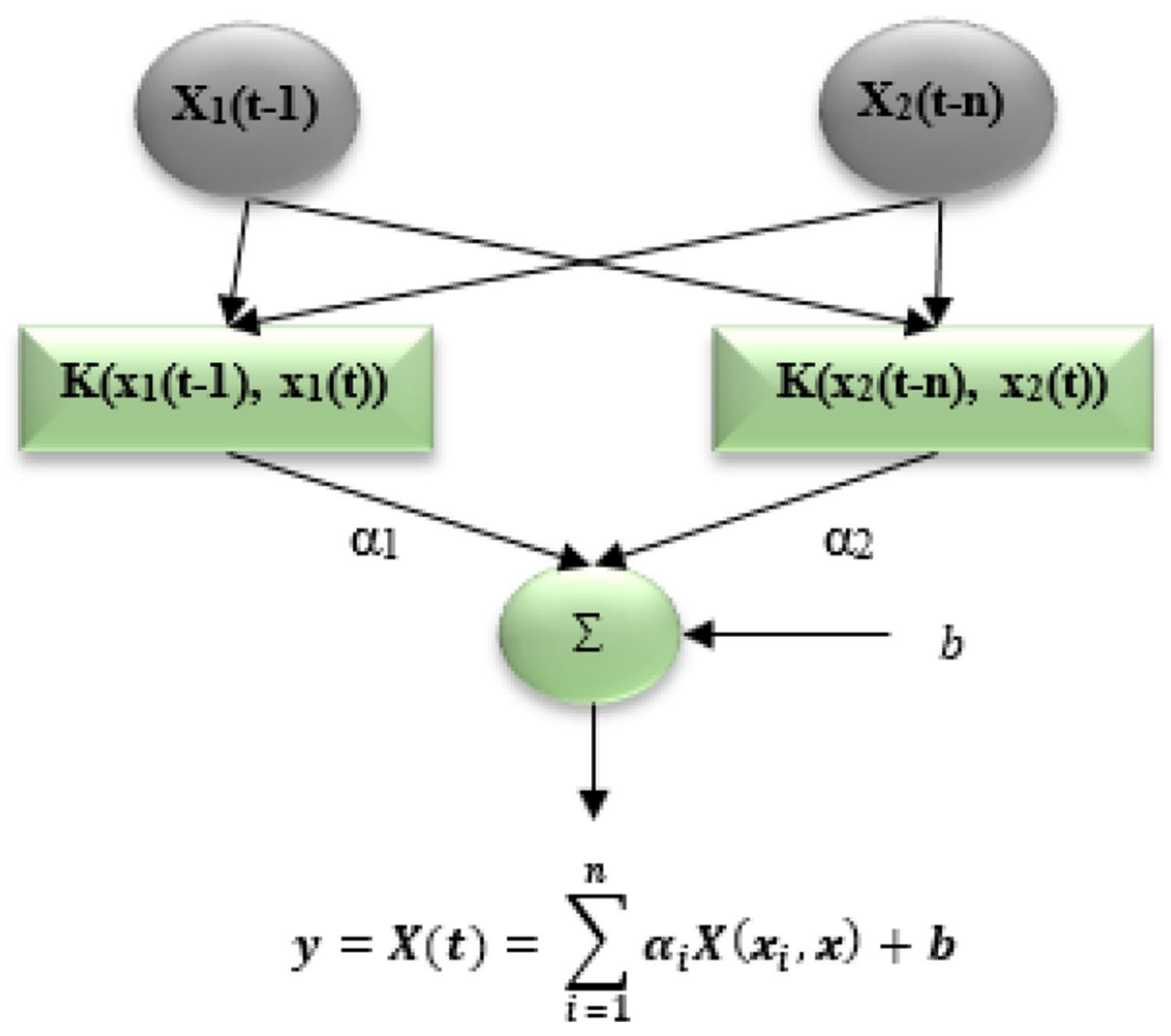

\subsubsection{Simple averaging ensemble (SAE)}

In proposed ensemble machine learning, as the name implies it involves taking the average of the outputs of the single models (HW, MLP and SVM). Initially, the simple models are calibrated and verified separately; consequently, the average results of HW, MLP and SVM was taken, which is then verified and compared with the experimental values. SAE can be expressed as follows;

$P_{(t)}=\frac{1}{N} \sum_{i=1}^{N} p_{i}(t)$

$N$ is denoted as the total number of the models (here $N=3)$ and $\mathrm{p}_{i}$ describes the result of each single model (i.e., HW, MLP, and SVM) during a time $t$.

\subsubsection{Support vector machine ensemble (SVM-E)}

Generally, in SVM-E method, the process is conducted through taking the average of the models non-linearly. Whereby, a new support vector machine (SVM) will be trained using the output results of the single models (MLP, HW and SVM) as the new input variables, Which will be simulated and then compared with the experimental values.

\subsection{Model validation and performance indices}

In any computational data-driven approach, the primary aim is to fit the models to a given data sets based on the employed indicators to produce a satisfactory prediction of the unknown data set. Considering issues such as overfitting, reliable training performance is not agreement with the verification performance always. In the validation process, different types of validation approach can be applied, including cross-validation, which is called k-fold cross-validation, others are holdout; leave one out, and so on. The holdout method is also considered as the simple version of $\mathrm{k}$-fold. Where the data is usually divided randomly into two sets known as the training and testing phases. The major advantages of the $k$ fold cross validation mechanism are that in every single round, the validation set and the training sets are independent [26]. As stated above, the data is further divided into categories $75 \%$ for the calibration (training) and $25 \%$ for the testing (verification) 
stage. Considering the fourfold cross-validation. It is very important to note that other validation methods can be applied to the data set [27].

In this work, the Nash-Sutcliffe efficiency (NSE) and correlation coefficient (CC) as goodness of fit and a statistical error inform of root mean-square error (RMSE), were used for the evaluation of the models:

$$
\begin{aligned}
& N S E=1-\frac{\sum_{j=1}^{N}\left[(Y)_{o b s, j}-(Y)_{c o m, j}\right]^{2}}{\sum_{j=1}^{N}\left[(Y)_{o b s, j}-\overline{(Y)}_{o b s, j}\right]^{2}} \\
& C C=\frac{\sum_{i=1}^{N}\left(Y_{o b s}-\bar{Y}_{o b s}\right)\left(Y_{c o m}-\bar{Y}_{c o m}\right)}{\sqrt{\sum_{i=1}^{N}\left(Y_{o b s}-\bar{Y}_{o b s}\right)^{2}} \sum_{i=1}^{N}\left(Y_{c o m}-\bar{Y}_{c o m}\right)^{2}}
\end{aligned}
$$

$$
R M S E=\sqrt{\frac{\sum_{i=1}^{N}\left(Y_{\text {obsi }}-Y_{\text {comi }}\right)^{2}}{N}}
$$

where $N, Y_{\text {obsi }}, \bar{Y}$ and $Y_{\text {comi }}$ are the data number, observed data, average value of the observed data and computed values, respectively.

\section{Results and discussion}

In the development of these models (MLP, HW and SVM), the simulation was done in MATLAB 9.3 (R2019a). For MLP model, a special algorithm known as Levenberg-Marquardt was used by employing 1000 iterations, coefficient of momentum of 0.9 , learning speed of 0.01 as well as an MSE of 0.0001 . Optimal finding of the parameters $(C \varepsilon, \gamma)$ is paramount in SVM model. The major reason for employing dataset completely outside the models is to ascertain the robustness and precision of the techniques employed during the simulation. The primary motivation for employing different data-intelligence models to different data set outside the models is attributed to the difficulty in understanding whether a specific model is superior to others in practice. Therefore, choosing appropriate models for a particular case can be challenging for modellers. This complexity can only be overcome by selecting and comparing different data-driven models, including the linear models despite their weaknesses in handling highly nonlinear and complex data. The correlation matrix of various variables used in this work is shown in Fig. 4. According to Gulbandilar et al. [28] correlation matrix indicated the relationship that exists between various parameters in this study. The strength of this relationship do not depend on the direction and sign. It can be seen from the correlation matrix
Fig. 4 Correlation of the variables

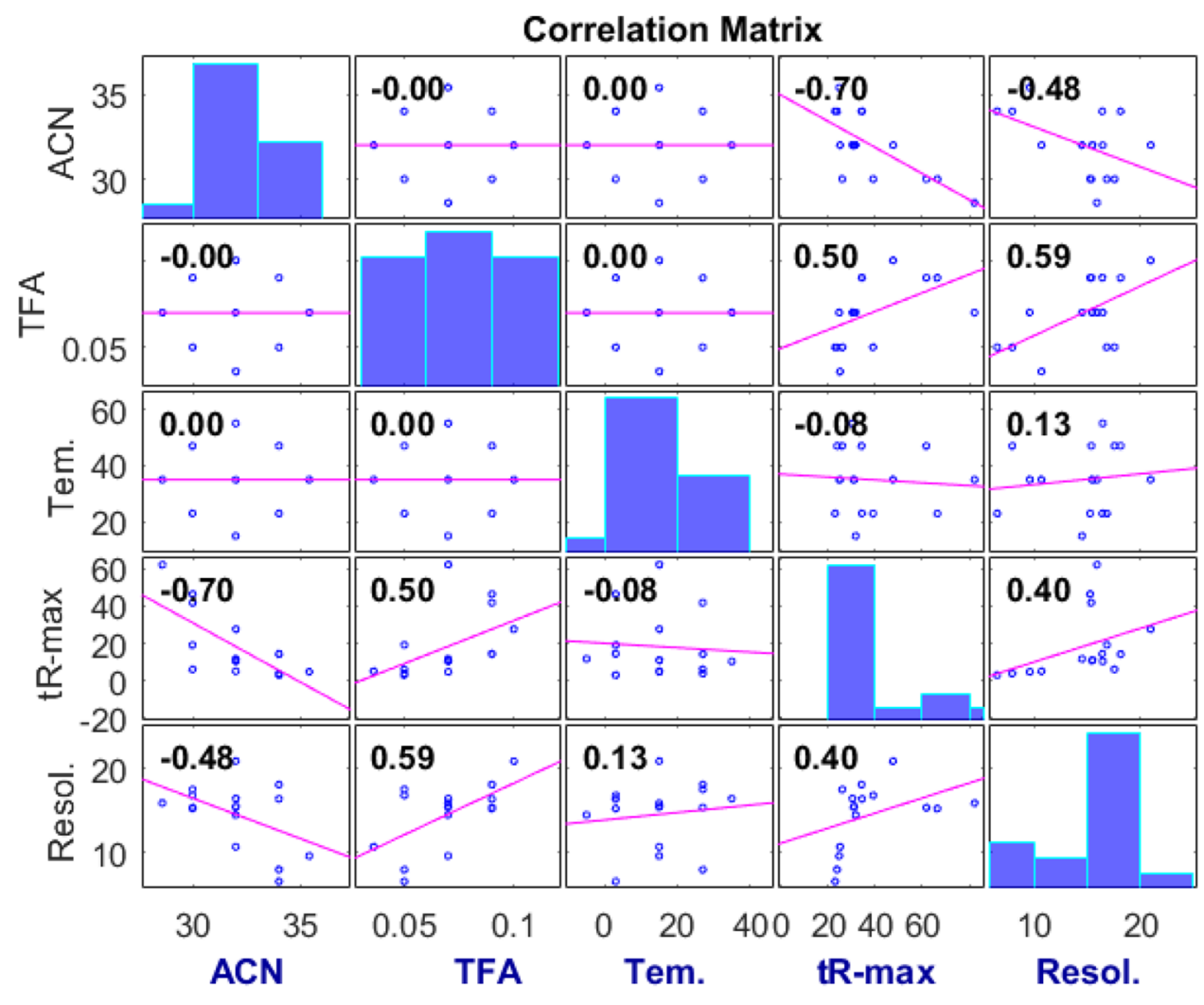


Table 1 Results of the single non-linear models

\begin{tabular}{|c|c|c|c|c|c|c|}
\hline & \multicolumn{3}{|c|}{ Training } & \multicolumn{3}{|l|}{ Testing } \\
\hline & NSE & RMSE & $\mathrm{CC}$ & NSE & RMSE & $\mathrm{CC}$ \\
\hline HW-tR max & 0.8139 & 8.2278 & 0.9022 & 0.8034 & 10.8730 & 0.8963 \\
\hline MLP-tR max & 0.8956 & 6.1624 & 0.9464 & 0.8673 & 8.9360 & 0.9313 \\
\hline SVM-tR max & 0.9632 & 3.6581 & 0.9814 & 0.9478 & 4.9837 & 0.9814 \\
\hline HW-Resol. & 0.8425 & 2.3140 & 0.9179 & 0.8240 & 4.5011 & 0.9078 \\
\hline MLP-Resol. & 0.9218 & 2.0499 & 0.9601 & 0.9042 & 3.3212 & 0.9509 \\
\hline SVM-Resol. & 0.9481 & 1.6698 & 0.9737 & 0.9386 & 2.6594 & 0.9688 \\
\hline
\end{tabular}

that TFA has the strongest correlation with the two outputs having $R=0.50$ with $t R-\max$ and $R=0.59$ with resolution.

\subsection{Artificial intelligence (AI) based models results}

The comparative predictive results of the Al-based models is demonstrated in Table 1. From the predictive results, it can be confidently said that all the three models are able of predicting the qualitative properties of the anti-Alzheimer agent. Among the Al-based models, SVM showed the highest performance skills as compared with MLP and HW models in simulating the tR-max and resolution of the anti-Alzheimer agent in both the training and testing phases based on Nash-Sutcliffe efficiency (NSE), correlation coefficient (CC) and a statistical error inform of root mean-square error (RMSE). Thus, the prediction performance accuracy based on Nash-Sutcliffe efficiency (NSE) indicated that SVM outperformed MLP and HW models and enhance their performance skills by 7 and $15 \%$ respectively in predicting the tR-max of the anti-Alzheimer agent in the testing stage. Further comparative analysis of the result demonstrated that SVM outperformed both MLP and HW models and boost their performance accuracy up to 3 and $12 \%$ respectively in predicting the resolution of the anti-Alzheimer agent in the testing stage.

The comparative predictive analysis of these models can be shown graphically using a scatter plot (see, Fig. 5). The scatter plot shows that there is higher agreement between the experimental and simulated values especially for SVM model.

Predictive comparative analysis of the models performance error is also demonstrated in Fig. 6. It is noteworthy to say that the errors in terms of RMSE are all within the acceptable range in both the training and testing stages. This showed that the models have the ability of capturing the complex and chaotic system for the simulation of the anti-Alzheimer agent. This study is also in agreement with other works conducted in the technical literature such as $[25,27]$.

The comparative analysis of the models was equally roved in various published articles in the technical literature as in [29-33]. However, the correlation co-efficient
(CC) depicted in Table 1 demonstrated the advantages of these non-linear models in humanin qualitative prediction. Figure 7 showed the performance of tR-max and resolution of the anti-Alzheimer agent using a radar surface chart in both the calibration and verification stages.

The comparative performance of the result using a radar plot in terms of correlation co-efficient (CC) are shown based on their increasing performance as follows: HW $(0.9022,0.8963), \operatorname{MLP}(0.9464,0.9313)$ and SVM $(0.9814,0.9814)$ in the calibration and verification stages respectively for modelling the $\mathrm{tR}$-max of the anti-Alzheimer agent. Whereas for the simulation of resolution, it is based on the following order: HW $(0.9179,0.9078)$, MLP $(0.9601,0.9509)$ and SVM $(0.9737,0.9688)$. Based on different studies in the literature, it is reported that radar scale ranges between $0-1$, whereby the best model result is close to 1 . The qualitative predictive performance skills of the models can be arranged in the following order $\mathrm{SVM}>\mathrm{MLP}>\mathrm{HW}$ for modelling both resolution and $\mathrm{tR}-\mathrm{max}$ of the anti- Alzheimer's agent in the HPLC optimization method development.

\subsection{The ensemble machine learning results}

Based on the comparative analysis of the models' accuracy, as indicated in Table 2 that both the two ensemble techniques are able to predict the qualitative performance of the anti-Alzheimer agent based on its tR-max and resolution as the corresponding output variables. The non-linear ensemble technique, SVM-E having NSE $=0.9995$ and 0.9994 in the both the training and testing stages proved to be superior over the linear ensemble technique SAE for simulating the qualitative properties of humanin and its derivatives. Moreover, close analysis of other performance indices RMSE as a statistical error and CC as a goodness of fits proved the reliability of SVM-E over SAE.

The predictive performance of the ensemble machine can be demonstrated using a scatter plot in order to determine how fit the simulated values were as compared with the experimental values. Figure 8 demonstrated the performance of both SVM-E and SAE graphically using a scatter plot. 

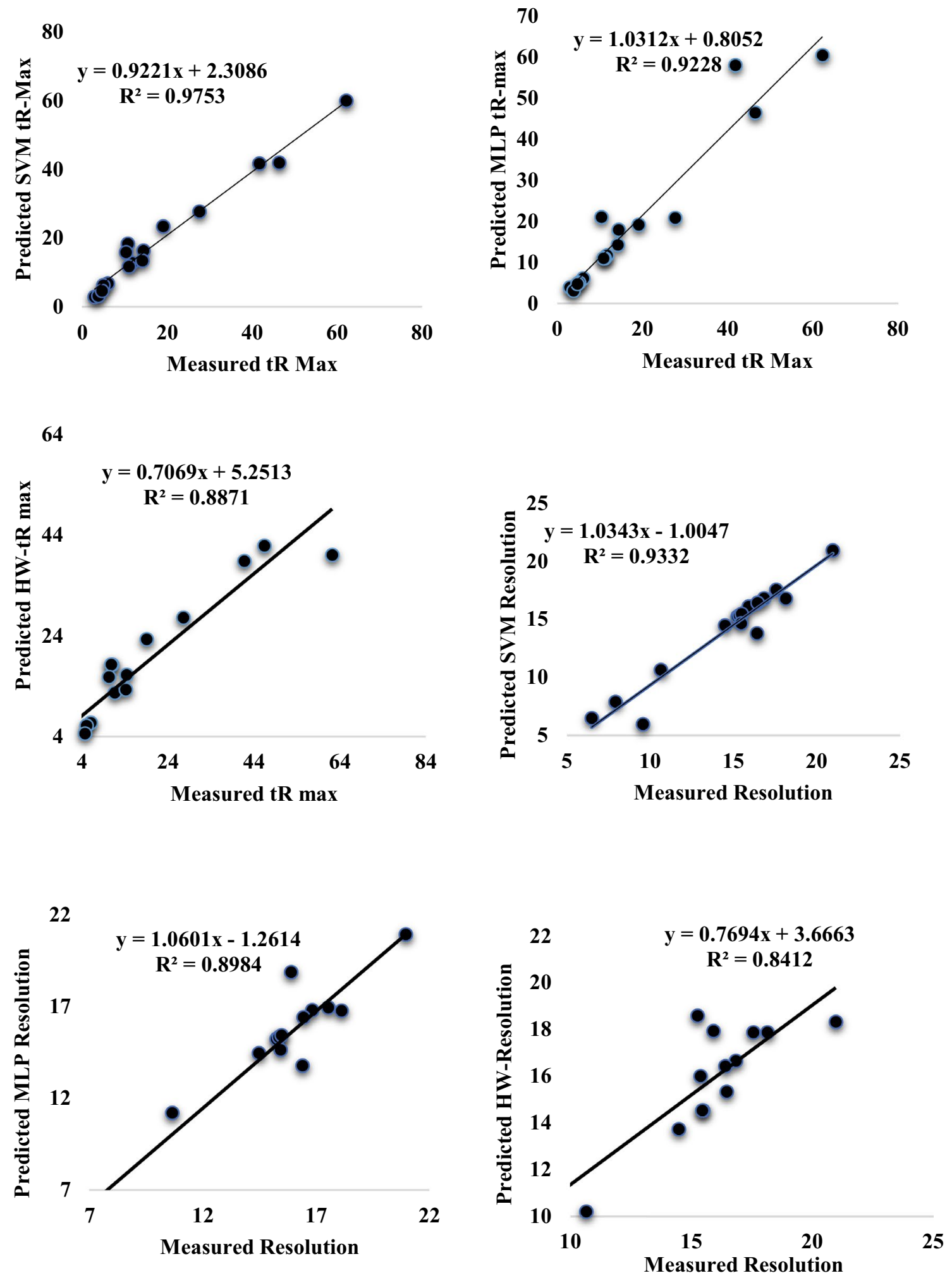

Fig. 5 Scatter plots representations of HW, MLP, and SVM for maximum retention time (tR-max) and resolution (Resol.) in modelling humanin 
Fig. 6 Comparative analysis of the performance error of the Al-based models

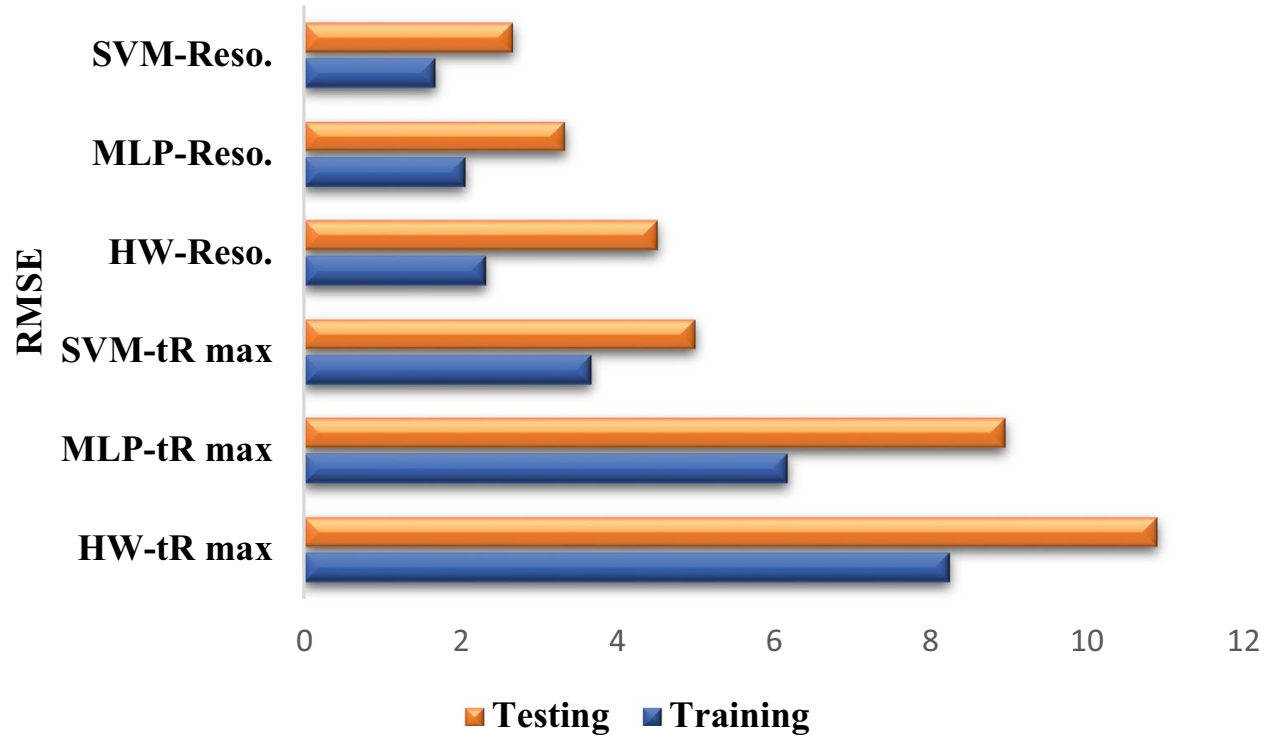

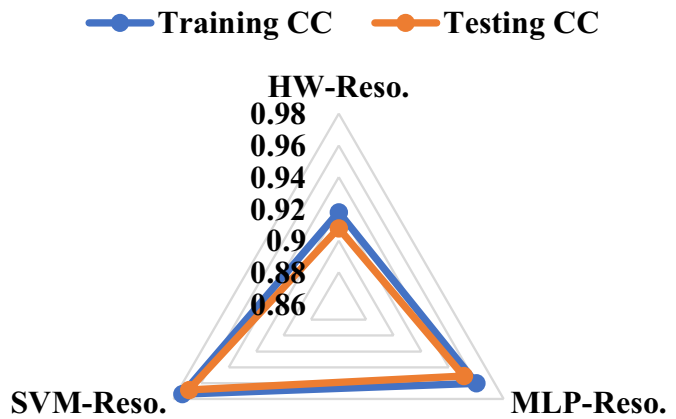

Fig. 7 Comparison of correlation co-efficient (CC) in terms Radar chart for tR-max and resolution in both the calibration and verification stages

Table 2 The ensemble machine learning results

\begin{tabular}{|c|c|c|c|c|c|c|}
\hline & \multicolumn{3}{|c|}{ Training } & \multicolumn{3}{|l|}{ Testing } \\
\hline & NSE & RMSE & $\mathrm{CC}$ & NSE & RMSE & $\mathrm{CC}$ \\
\hline SVM-E-tR max & 0.9995 & 0.4271 & 0.9997 & 0.9994 & 0.2688 & 0.9997 \\
\hline SAE-tR max & 0.9700 & 3.3029 & 0.9849 & 0.9687 & 1.0829 & 0.9951 \\
\hline SVM-E-Resol. & 0.9994 & 0.1836 & 0.9997 & 0.9992 & 0.2308 & 0.9996 \\
\hline SAE-Resol. & 0.9816 & 0.9953 & 0.9907 & 0.9797 & 1.1845 & 0.9898 \\
\hline
\end{tabular}

The ensemble machine learning techniques' comparative analysis results can also be compared graphically using radar chart (see Fig. 9). The main aim of using the radar chart is to display the multivariate quantitative analysis. It equally demonstrates the highest and lowest scoring variables within a data set, and this lead to flexible comparative performance. From the radar chart it can be observed that SVM-E has shown higher performance as compared with the SAE ensemble technique for modelling the qualitative properties of the anti-Alzheimer agent. This is in line with Table 2.

The predictive performance of the ensemble machine learning technique in terms of NSE showed that SVM-E outperformed SAE and increased its performance skills up to $3 \%$ and $2 \%$ in the modelling of $\mathrm{tR}$-max and resolution respectively in the verification stage. SVM-E can further be compared with the single non-linear models in order to demonstrate the robustness and satisfactory of the 

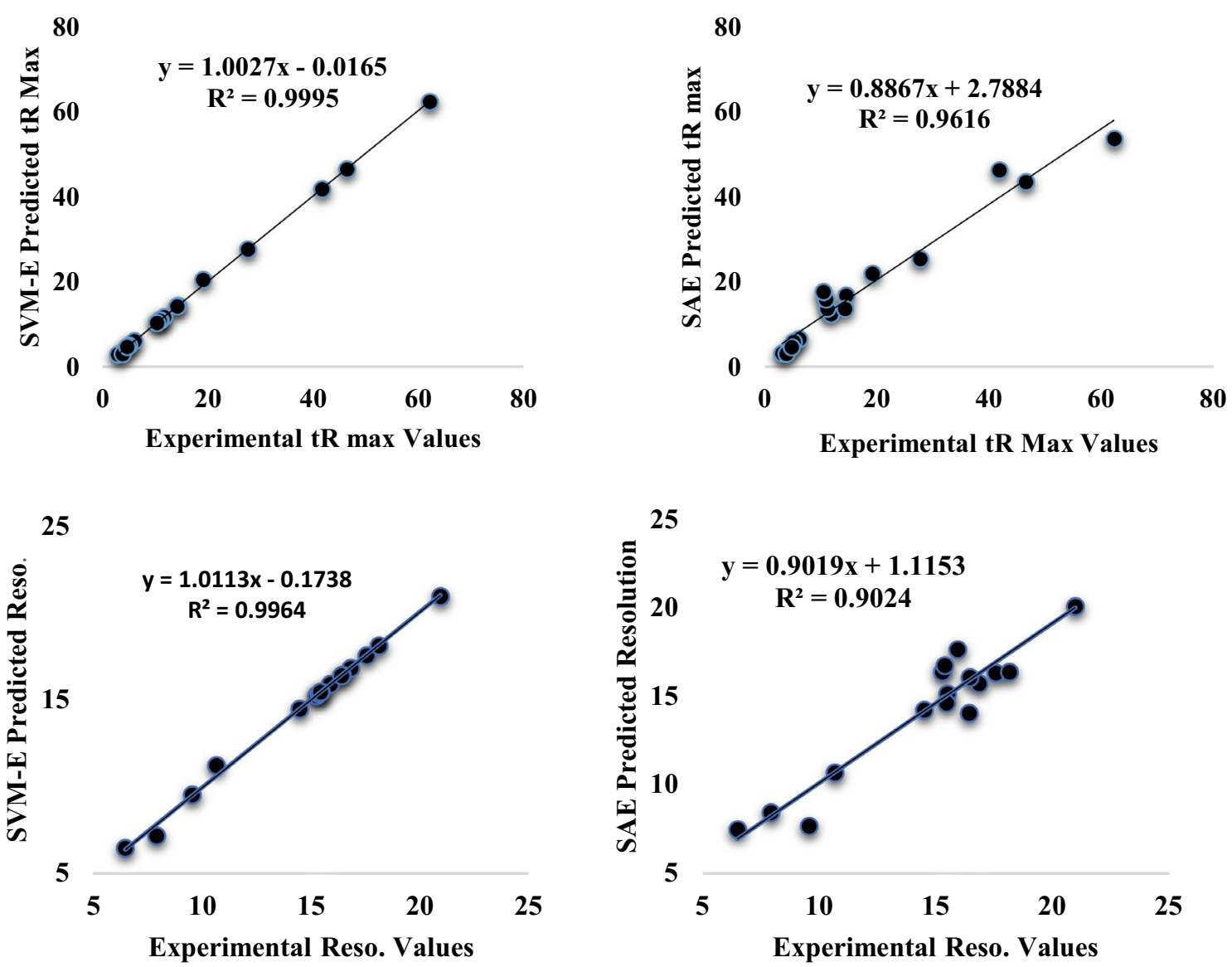

Fig. 8 Scatter plots of the ensemble machine learning for both tR-max and resolution

ensemble machine learning method. The comparative performance of SVM-E inform of NSE indicated its ability in boosting and enhancing the performance skills of the

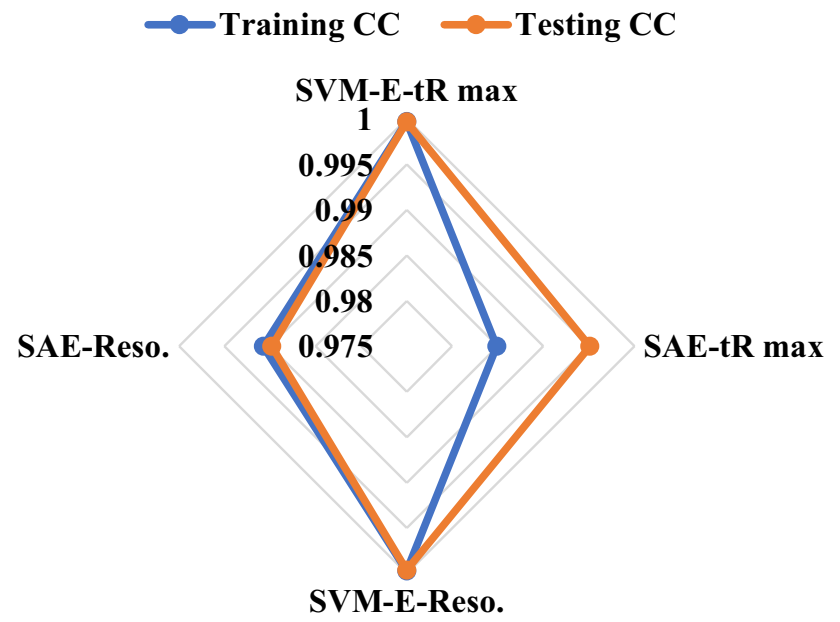

Fig. 9 Radar plot of the ensemble machine learning in terms of CC for the qualitative properties of humanin and its derivatives in both the training and testing stages single models SVM, MLP and HW models up to $5 \%, 13 \%$ and $20 \%$ respectively in the testing stage for modelling tR-max.

\section{Conclusion}

This study employs the application of artificial intelligence (Al) based models including; multi-layer perceptron (MLP), Hammerstein-Wiener (HW) and support vector machine (SVM) for the qualitative prediction of anti-Alzheimer agent. Mobile phases (in form of ACN and TFA) and column temperature are employed as the predictors. In this research, the data was obtained from previous experimental designed published in the technical literature as discussed in chapter 2 . The quantitative performance indicated that SVM outperformed MLP and HW models and increases their performance accuracy by 7 and $15 \%$ respectively in predicting the tR-max of the anti-Alzheimer agent in the testing stage. Further comparative analysis of the result demonstrated that SVM outperformed both MLP and HW models and boost their performance accuracy up 
to 3 and $12 \%$ respectively in predicting the resolution of the anti-Alzheimer agent in the testing stage.

Observation of different outputs of the Al-based models at various time intervals showed the necessity of ensembling the outputs of the Al-based models. Thus, a nonlinear ensemble technique inform of SVM-E and a linear ensemble technique inform of SAE were employed to enhance the performance accuracy of the single models. The predictive performance of the ensemble machine learning technique in terms of NSE showed that SVM-E outperformed SAE and increased its performance skills up to 3 and $2 \%$ in the modelling of tR-max and resolution respectively in the verification stage. SVM-E can further be compared with the single non-linear models in order to demonstrate the robustness and satisfactory of the ensemble machine learning method. The comparative performance of SVM-E inform of NSE indicated its ability in boosting and enhancing the performance skills of the single models SVM, MLP and HW models up to 5, 13 and $20 \%$ respectively in the testing stage for modelling tR-max.

\section{Compliance with ethical standards}

Conflict of interest The authors acknowledged that there is no potential conflict of interest that can hinder the submission of this work.

\section{References}

1. Snyder LR, Dolan JW (2007) High-performance gradient elution: the practical application of the linear-solvent-strength model. Wiley, Hoboken

2. Munro K, Miller TH, Martins CPB, Edge AM, Cowan DA, Barron LP (2015) Artificial neural network modelling of pharmaceutical residue retention times in wastewater extracts using gradient liquid chromatography-high resolution mass spectrometry data. J Chromatogr A 1396:34-44

3. Shojaeimehr T, Rahimpour F (2018) Retention time modelling of short-chain aliphatic acids in aqueous ion-exclusion chromatography systems under several conditions using computational intelligence methods (artificial neural network and adaptive neuro-fuzzy inference system). J Liq Chromatogr Relat Technol 41(12):810-817

4. Chen H, Poon J, Poon SK, Cui L, Fan K, Yuen Sze DM (2015) Ensemble learning for prediction of the bioactivity capacity of herbal medicines from chromatographic fingerprints. BMC Bioinf 16(12):S4

5. Liu $Y$ et al (2019) Artificial intelligence-based breast cancer nodal metastasis detection insights into the black box for pathologists. Arch Pathol Lab Med 143(7):859-868

6. D'Archivio AA (2019) Artificial neural network prediction of retention of amino acids in reversed-phase HPLC under application of linear organic modifier gradients and/or $\mathrm{pH}$ gradients. Molecules 24(3):632

7. Novotná K, Havliš J, Havel J (2005) Optimization of high performance liquid chromatography separation of neuroprotective peptides: fractional experimental designs combined with artificial neural networks. J Chromatogr A 1096(1-2):50-57

8. Veenaas C, Linusson A, Haglund P (2018) Retention-time prediction in comprehensive two-dimensional gas chromatography to aid identification of unknown contaminants. Anal Bioanal Chem 410(30):7931-7941

9. Shao C et al (2018) Ridgetail white prawn (Exopalaemon carinicauda) $\mathrm{K}$ value predicting method by using electronic nose combined with non-linear data analysis model. Food Anal Methods 11(11):3121-3129

10. Abba SI, Usman AG, Işik S (2020) Simulation for response surface in the HPLC optimization method development using artificial intelligence models: a data-driven approach. Chemom Intell Lab Syst 201:104007

11. Choubin B, Khalighi-Sigaroodi S, Malekian A, Kişi Ö (2016) Multiple linear regression, multi-layer perceptron network and adaptive neuro-fuzzy inference system for forecasting precipitation based on large-scale climate signals. Hydrol Sci J 61(6):1001-1009

12. Kim S, Singh VP (2014) Modeling daily soil temperature using data-driven models and spatial distribution. Theor Appl Climatol 118(3):465-479

13. Pham QB et al (2019) Potential of hybrid data-intelligence algorithms for multi-station modelling of rainfall. Water Resour Manag 33(15):5067-5087

14. Committee AT (2000) Artificial neural networks in hydrology. I: preliminary concepts. J Hydrol Eng 5(2):115-123

15. Kim JH, Lee HS (2017) Improvement of early strength of cement mortar containing granulated blast furnace slag using industrial byproducts. Materials (Basel) 10(9):1050

16. Gaya MS et al (2017) Estimation of turbidity in water treatment plant using Hammerstein-Wiener and neural network technique. Indones J Electr Eng Comput Sci 5(3):666-672

17. Abba SI, Nourani V, Elkiran G (2019) Multi-parametric modeling of water treatment plant using Al-based non-linear ensemble. J Water Supply: Res Technol-Aqua 2:1-15

18. Abba SI, Saleh A, Hamza N, Tukur Al, Wahab NA (2019) Modelling of uncertain system : a comparison study of linear and non-linear approaches. IEEE

19. Abba SI et al (2020) Emerging evolutionary algorithm integrated with kernel principal component analysis for modeling the performance of a water treatment plant. J. Water Process Eng. 33:101081

20. Vapnik V (1995) The nature of statistical learning theory, p 188.

21. Granata F, Papirio S, Esposito G, Gargano R, de Marinis G (2017) Machine learning algorithms for the forecasting of wastewater quality indicators. Water (Switzerland) 9(2):1-12

22. Baba NM, Makhtar M, Abdullah S, Awang MK (2015) Current issues in ensemble methods and its applications. J Theor Appl Inf Technol 81(2):266-276

23. Loos S, Shin CM, Sumihar J, Kim K, Cho J, Weerts A (2019) Ensemble data assimilation methods for improving river water quality forecasting accuracy. Water Res 171:115343

24. B FA, Sadaoui $S$ (2019) Multi-class ensemble learning. Springer

25. Dehghanian E, Kaykhaii M, Mehrpur M (2015) Comparison of single best artificial neural network and neural network ensemble in modeling of palladium microextraction. Mon Chem 146(8):1217-1227

26. Usman AG, Işik S, Abba SI (2020) A novel multi-model datadriven ensemble technique for the prediction of retention factor in HPLC method development. Chromatographia 83:933-945

27. Yaseen ZM et al (2019) A novel hybrid evolutionary data-intelligence algorithm for irrigation and power production management: application to multi-purpose reservoir systems. Sustainability 11(7):1953 
28. Gulbandilar E, Kocak Y (2013) Prediction of the effects of fly ash and silica fume on the setting time of Portland cement with fuzzy logic. Neural Comput Appl 22(7-8):1485-1491

29. Ramzi M, Kashaninejad M, Salehi F, Sadeghi Mahoonak AR, Ali Razavi SM (2015) Modeling of rheological behavior of honey using genetic algorithm-artificial neural network and adaptive neuro-fuzzy inference system. Food Biosci. 9(1):60-67

30. Arabameri M, Nazari RR, Abdolshahi A, Abdollahzadeh M, Mirzamohammadi S, Shariatifar N, Barba FJ, Khaneghah AM (2019) Oxidative stability of virgin olive oil: evaluation and prediction with an adaptive neuro-fuzzy inference system (ANFIS). J Sci Food Agric 99(12):5358-5367

31. Alsharksi AN, Danmaraya YA, Abdullahi HU, Ghali UM, Usman AG (2020) Potential of hybrid adaptive neuro fuzzy model in simulating clostridium difficile infection status. Int J Basic Appl Sci 3(1):1-6
32. Ghali UM, Usman AG, Degm MAA, Alsharksi AN, Naibi AM, Abba SI (2020) Applications of artificial intelligence-based models and multi-linear regression for the prediction of thyroid stimulating hormone level in the human body. Int J Adv Sci Technol 29(4):3690-3699

33. Abdullahi HU, Usman AG, Abba SI (2020) Modelling the absorbance of a bioactive compound in HPLC method using artificial neural network and multilinear regression methods. Dutse J Pure Appl Sci (DUJOPAS) 6(2):362-371

Publisher's Note Springer Nature remains neutral with regard to jurisdictional claims in published maps and institutional affiliations. 